\title{
SocArXiv
}

Preprint : 22 April 2021

10.31235/osf.io/sbwcq

\section{Introducing Preregistration of Research Design to Archaeology}

\author{
Shawn A. Ross \\ Macquarie University
}

\author{
Brian Ballsun-Stanton \\ Macquarie University
}

\begin{abstract}
Archaeology has an issue with "just-in-time" research, where insufficient attention is paid to articulating a research design before fieldwork begins. Data collection, management, and analysis approaches are under-planned and, often, evolve during fieldwork. While reducing the amount of preparation time for busy researchers, these tendencies reduce the reliability of research by exacerbating the effects of cognitive biases and perverse professional incentives. They cost time later through the accrual of technical debt. Worse, these practices hinder research transparency and scalability by undermining the quality, consistency, and compatibility of data. Archaeologists would benefit from embracing the "preregistration revolution" sweeping other disciplines. By publicly committing to research design and methodology ahead of time, researchers can produce more robust research, generate useful and reusable datasets, and reduce the time spent correcting problems with data. Preregistration can accommodate the diversity of archaeological research, including quantitative and qualitative approaches, hypothesis-testing and hypothesis-generating research paradigms, and place-specific and generalizing aims. It is appropriate regardless of the technical approach to data collection and analysis. More broadly, it encourages a more considered, thoughtful approach to research design. Preregistration templates for the social sciences can be adopted for use by archaeologists.
\end{abstract}

Keywords: archaeology, preregistration, open data, research design, research transparency.

\section{Cite this Preprint}

Ross, Shawn A., and Brian Ballsun-Stanton. Accepted 2 April 2020. "Introducing Preregistration of Research Design to Archaeology." In Digital Heritage and Archaeology in Practice, edited by Ethan Watrall and Lynne Goldstein. Gainesville, FL: University Press of Florida. 
Preregistration of research design, and in some cases hypotheses, has been promoted as a technique to combat researchers' biases, overcome perverse professional incentives, improve transparency, and increase rigor. The motivation stems from the "reproducibility crisis" faced by many disciplines (Nature 2016; Open Science Collaboration 2015; Adam 2019; Kaplan and Irvin 2015; Franco et al. 2014; Mellor et al. 2016; Baker 2015; Munafò et al. 2017; National Academies of Sciences, Engineering, and Medicine et al. 2019). Preregistration can be described as the declaration of a research plan before data collection begins (Center for Open Science 2018), or a process in which researchers: "define the research questions and analysis plan before observing the research outcomes" (Nosek et al. 2018a). Although often associated with scientific research, many disciplines can benefit from preregistration. Preregistration accommodates inductive (hypothesis-generating; postdictive) as well as deductive (hypothesis testing; predictive) research, idiographic (distinct to a particular place) as well as nomothetic (applicable to a larger class of events of conditions) approaches, qualitative as well as quantitative analyses, and can be applied at various stages of the research lifecycle (Nosek et al. 2018a). In archaeology and other field sciences, preregistration offers its usual benefits: explicit recognition of research design; management of biases and perverse incentives; and exposure of research methods and processes as required by emerging good practice in research transparency (Center for Open Science 2019).

Preregistration could also counteract a sociotechnical problem hindering the adoption of digital field methods: a reluctance to invest time and resources in the early planning and preparation phases of a project, versus time later during fieldwork, post-processing, and analysis (Sobotkova et al. 2016). This lack of planning leads to changes in research design during execution with little accountability, including for example, ongoing modification of data structures and data capture workflows during fieldwork (Borgman 2015). Such in-progress changes raise the cost and complexity of digital field data collection systems. Ad hoc changes limit data quality by precluding adequate testing and refinement, undermining consistency of data and methods, and imposing "technical debt" such as extensive cleaning and reconciliation of data at the end of the project. These "easy" changes during and after fieldwork thereby hinder the interoperability and reusability of resulting datasets.

This chapter will explore how preregistration can benefit domains like archaeology that involve approaches spanning the humanities and the sciences, with both deductive and inductive elements. We discuss the benefits of preregistration for archaeological research, argue that preregistration is feasible for archaeologists, and propose how preregistration might be implemented based on a model from qualitative research in the social sciences.

\section{Preregistration Accommodates Predictive and Postdictive Approaches}

Preregistration has been promoted as a way to improve confidence in the results of research, especially by clarifying whether research generates new hypotheses from existing data, or tests existing hypotheses using new data. Maintaining this distinction combats researchers' cognitive biases, ensures that research adheres to assumptions intrinsic to common statistical approaches, and counteracts the distortions introduced by perverse professional incentives that encourage questionable research practices. For example, we can see a "publication bias" towards papers that confirm, rather than refute, initial hypotheses in deductive research (Chase 2013; Fraser et al. 2018). Although preregistration is often associated with scientific and biomedical research (https://clinicaltrials.gov/ is the largest existing registry), it is also used in social 
sciences like economics and political science (see https://www .socialscienceregistry.org/; http://egap.org/content/registration/; cf. Nosek et al. 2018a; Haven and Van Grootel 2019).

Preregistration emerged as a new methodological commitment in disciplines like psychology and biomedicine that have struggled to replicate or reproduce results. The problems it addresses, however, exist in many domains. Nosek, for example, emphasizes researchers' tendency to conflate "prediction" and "postdiction". Prediction describes a deductive, hypothesis-testing approach since the hypothesis predicts outcomes of empirical research. Postdiction describes an inductive, hypothesis-generating approach since the hypothesis arises from (and after) empirical research (Nosek et al. 2018a; Haven and Van Grootel 2019). Predictive research provides a rigorous way to test and disprove hypotheses. Such testing is a boon for positivists, who remind us that Popper's injunction that "proof" of a hypothesis is always provisional while disproof is final, making falsifiability the hallmark of science (Derksen 2019). Postdictive research, conversely, can generate surprising or unexpected hypotheses that radically shift our understanding of the world or even change scientific worldviews (Kuhn 1970:23).

Both predictive and postdictive approaches are essential, but they must not be conflated. Therefore, even if a researcher does not hold with positivist Popperian falsification as the primary mechanism for scientific discovery (Huemer 2020), formalisms like preregistration that enforce distinctions between prediction and postdiction improve the quality of research:

"Failing to appreciate the difference can lead to overconfidence in post hoc explanations (postdictions) and inflate the likelihood of believing that there is evidence for a finding when there is not. Presenting postdictions as predictions can increase the attractiveness and publishability of findings by falsely reducing uncertainty" (Nosek et al. 2018a).

If postdiction is conflated with prediction, it is prone to "fallibility of memory, motivated reasoning, and cognitive biases" (Nosek et al. 2018a). Of particular concern is hindsight bias, where researchers (often subconsciously) remember or seek evidence supporting their conclusions while ignoring or faulting contrary evidence. Since common statistical methods like null hypothesis significance testing, moreover, assume prediction rather than postdiction, they are likely to produce "Type 1 errors" (false positives) when researchers neglect the difference. As a result of these problems, "Mistaking postdiction as prediction underestimates the uncertainty of outcomes and can produce psychological overconfidence in the resulting findings" (Nosek et al. 2018a). Although postdictive research can produce new inconvenient facts that contradict received paradigms, it should not simultaneously test the hypotheses it generates.

Indeed, "using current results to construct post hoc hypotheses that are then reported as if they were a priori hypotheses", "failing to report a priori hypotheses that are unsupported by the current results" (Rubin 2017), or "presenting exploratory work as though it was confirmatory hypothesis testing" (Fraser et al. 2018), is considered "hypothesizing after the results are known" or HARKing (Kerr 1998). HARKing is always a questionable research practice if it is unreported, although disagreement exists about the acceptability (or even desirability) of careful and transparent post hoc analysis in deductive research (Rubin 2017; Hollenbeck and Wright 2017). In a 2018 paper surveying over eight hundred ecologists and evolutionary biologists, 51\% admitted to HARKing (Fraser et al. 2018). Fraser also notes in passing 
that when published papers fail to disclose a priori hypotheses (or if there were a priori hypotheses), it becomes difficult to judge whether HARKing - or the conflation of postdiction and prediction more broadly - has even taken place. As such, the a priori articulation of hypotheses required by preregistration (or the explicit statement that research is inductive) can help to combat this species of questionable research practice.

\section{The Problem of "Just-in-Time" Archaeology}

The biases and perverse incentives that preregistration was designed to combat in other disciplines also exist in archaeology. Processual archaeology's "scientific approach", with its emphasis on revealing aims and biases and its problem-orientation, has recognized these problems for half a century (e.g. Johnson 2010:24-25). Indeed, the relevance of early processual thought to the current problem is striking. In the 1970s, Hole criticized archaeologists' tendency to focus on fieldwork and analytical methods rather than research design, especially the development of hypotheses or models. He called for more "thought" before the archaeologist turned to "methodological manipulations" (Hole 1973:32). In the same proceedings, French observed that:

"[I]t is essential and (?) obligatory to define the problems before developing or choosing the means used to collect the data necessary to examine these problems and the hypotheses explicit in them. In other words, the questions asked dictate the methods of recovery; one selects the recovery technique to suit the problem in hand" (French 1973:105).

French went on to emphasize the importance of what would now be termed data provenance. Data provenance documents where material was found or observations made, by what method, under what conditions, and with what sampling approach. He further recommended that archaeologists specify the program and standards governing data collection. Only by doing so can the quantity, quality, and comparability of archaeological data be assessed. The situation French described - a lack of shared (or at least articulated) standards of data acquisition and documentation - remains widespread today, undermining the comparability of data from different projects (French 1973:106; Atici et al. 2013; Faniel et al. 2013; Holub et al. 2018). Influential recoomendations, such as those encapsulated by Findable, Accessible, Interoperable, and Reusable (FAIR) data and the Transparency and Openness Promotion (TOP) Guidelines likewise emphasize the creation of proper metadata - the documentation of data such that it can be understood and reused by outsiders (Wilkinson et al. 2016; Center for Open Science 2019). In short, merely collecting more data does not automatically support the syntheses needed to produce a better understanding of the past, and data-recovery techniques must harmonize with theoretical approaches (e.g. Johnson 2010:24-25; French 1973:107). Preregistration encourages the planning and preparation necessary to collect data appropriate to a project's aims now and useful to others in the future.

Preregistration can, moreover, help researchers articulate their specific theoretical approach and research tradition. Processualism and post-processualism both have fundamental assumptions about the nature of evidence, approaches to knowledge, and the interpretation of data. They are examples of competing Lakatosian research programs, each with their own "hard-core" of truths which they take to be self-evident and "soft outer shell" of current, unproven, research 
questions (Lakatos 1978). Preregistration makes such researcher assumptions more explicit (Johnson 2010:3).

Preregistration also mitigates a specific sociotechnical barrier to the adoption of digital approaches in archaeology. Archaeologists tend to substitute remedial work at the end of a project for proper planning and preparation at the beginning (Sobotkova et al. 2016). This tendency, along with the continuing prevalence of print publication of datasets like artifact catalogs, impedes the production of comprehensive, reusable datasets in archaeology. It also undermines the cultivation of deeper digital practice more generally.

This sociotechnical barrier to innovation in the discipline warrants further discussion. Our information infrastructure work, related to the Field Acquired Information Management Systems (FAIMS) project, has customized field data collection systems for over sixty workflows at more than forty projects in archaeology, ecology, geoscience, history, and other disciplines since 2014 (Ross et al. 2013, 2015; Sobotkova et al. 2015, 2016; Ballsun-Stanton et al. 2018; VanValkenburgh et al. 2018). We have found that field researchers broadly, and archaeologists particularly, under-invest in planning and preparation in three ways.

First, archaeologists tolerate a lack of detail or precision in their documentation. For example, they do not take the time to build and deploy controlled vocabularies or they leave important and recurring information for "notes" or other free-text fields. When applied to paper-based recording, these practices exploit the forgiving nature of the medium, relying on the ability to write "in the margins" or "on the back of the page". In digital recording, these tendencies often translate into poorly designed and unvalidated spreadsheets or databases. This haste and imprecision reduces the up-front investment in data and workflow modeling for harried academics and consultants but adds an implicit cost: a much larger burden of post-fieldwork data cleaning.

Second, forms and protocols assume a great deal of implicit knowledge transmitted orally, often informally, at the project. This practice reduces the transparency of fieldwork and fails to record important metadata needed later for data reuse. Implicit methods and metadata are lost by team members, volunteers, and students who forget just how things were done, are uncontactable, or who have moved onto other projects. This epistemic turnover impedes later understanding and reuse by outsiders - or even project participants - who cannot retrieve lost context.

Third, project leaders tend to develop record-keeping or documentation practices like forms and protocols late, shortly before fieldwork begins, leaving insufficient time to test and refine them with realistic trial data. This practice exacerbates the effects of the first two problems, producing records that only approximate the documentation that researchers desire. It also contributes to a feature common to many fieldwork-based domains, a tendency for research design to be "emergent" - for researchers to elaborate and modify approaches, methods, tools, technologies, and practices during fieldwork when working with real data (Borgman 2015:106-107). Although a certain amount of "tinkering" (c.f. Sobotkova and Janouchova 2021, Chapter 6 this volume) may be necessary to mitigate the unpredictability of fieldwork, the practice produces datasets that are not even consistent or comparable across a single season at a single project without considerable manual reconciliation, let alone more broadly.

Together, these practices constitute a sociotechnical hurdle to effective digital archaeology: underinvestment in the early stages of a project and subsequent accrual of technical debt, requiring the expenditure of much more time and effort to "fix" data at the end of a project 
(Sobotkova et al. 2016). This misallocation of time extends to - and is exacerbated by - an under-allocation of research funds to data management (Mons 2020). In data quality and reusability, as much as in analytical reproducibility, such "bolt-on" fixes applied at the end of a project are much more costly than "built-in" good practice, if they are feasible at all (Marwick 2017b). Perhaps of greatest concern, late data cleaning is often "lossy", irreversibly reducing the quality and utility of a project's data, a serious consideration due to the unrepeatable nature of most archaeological research.

Addressing this sociotechnical problem by improving research design, data production, and the relationship between them is crucial. The necessity of producing quality, consistent, reusable data is now recognized in archaeology and beyond. Data is becoming a first-class output and a key element of academic rigor. Publication of analytical code is likely to follow. Pressure is growing to improve the transparency of research by, for example, making data and other digital research objects FAIR (Wilkinson et al. 2016; GO-FAIR 2017; Stall et al. 2019). That pressure includes requirements established by journals, funders, and regulators, as well as broader pressure to implement better practice in research. For example, sharing of FAIR data has been written into the (Australian) National Statement on Ethical Conduct in Human Research and the Australian Research Council's Open Access Policy, both promulgated by the National Health and Medical Research Council (2018, 2019). Publishers representing over a thousand journals and many private funders have endorsed the TOP Guidelines (Mellor et al. 2016; Center for Open Science 2019). Meeting such requirements will require a greater investment, both in time and resources, from researchers in early planning and preparation. In the future, stakeholders will expect archaeologists' data and code, not just our results and conclusions. At present, however, data repositories are underpopulated and datasets are not routinely reused (McNutt et al. 2016; Sobotkova 2018). This underpopulation inhibits reproduction or verification of results, independent analyses of primary data, and the application of new techniques to old datasets. It also prevents the combination of datasets from multiple studies for large-scale research to address "grand challenges" (Snow et al. 2006; Kintigh 2006; Kintigh et al. 2014; McNutt et al. 2016).

\section{Preregistration is Suitable for Archaeology}

Preregistration can help resist the temptation of "just-in-time" research design. It would make "built-in" good practice more likely, as opposed to more common attempts to "bolt-on" fixes after fieldwork (Marwick 2017b:441). Specifically, preregistration offers a mechanism to make approaches and assumptions explicit, allowing them to be scrutinized (Johnson 2010:3) and counteracting cognitive biases. Preregistration also helps researchers overcome the sociotechnical tendency to underinvest in preparation for data acquisition and analysis, instead of relying on remediation of problems late in the research lifecycle. Combined with a commitment to other aspects of reproducibility (Perkel 2018; Marwick 2017b; Wilkinson et al. 2016), preregistration holds the potential to improve research practice tangibly in our discipline.

Preregistration can, moreover, accommodate the diversity and transdisciplinarity of archaeological research. Archaeology can be deductive and predictive or inductive and postdictive. Most often, archaeology - like other fieldwork disciplines - might best be described as abductive (Tavory and Timmermans 2014). Abductive research represents a synthesis of deductive and inductive approaches, gathering data according to a specific and intentional methodology 
(the inductive element), and then applying a theory or framework that describes those facts (deductive). Used in interpretive social science research, this methodology is seldom explicitly acknowledged or described (Lewis-Beck et al. 2003:1). Archaeology can also be quantitative and statistical or qualitative and descriptive. It can be idiographic, seek an in-depth understanding of the specific, contingent, and unique in a particular place and time. Or, it can be nomothetic, attempting to derive or test general (if provisional or incomplete) principles or rules by looking at similarities across space and time. Idiographic research is often associated with inductive and qualitative approaches in the humanities. Nomothetic research is usually associated with deductive and quantitative approaches in the social and natural sciences. These approaches, however, can cut across disciplines and can be combined, iterated, or synthesized in various ways (Watson 1973:51). A preregistration regime does not privilege any of these approaches over another but instead demands an articulation of, and a public commitment to, a specific research design at the beginning of a project, before research begins. Indeed, the very complexity and diversity of archaeology - spanning disciplines and approaches to knowledge - makes articulation of research design particularly important. An explicit research design documented in detail before research starts, rather than during the writing-up stage, avoids the unintentional conflation or elision of approaches that could undermine research results.

Archaeology is also serendipitous. Often, we do not know what we will find until we get to the field. This problem, however, is not unique to archaeology. Even in deductive disciplines, results are not and should not be under the control of the researcher (Chambers 2019). Research design, research questions or hypotheses, methods, and interpretive frameworks, however, are under researcher control. As a result, these elements can be articulated beforehand. Training, prior fieldwork, knowledge of analogous sites or landscapes, regulator or funder requirements, and disciplinary expectations around fieldwork and publication all inform research. These prior requirements and similarities allow archaeologists to state why and how they plan to undertake research, if not what they will find. Preregistration forces a level of clarity and formalizes an a priori commitment to research design and recording strategy. Indeed, since expected outcomes of research vary, and are often externally dictated (e.g., by heritage legislation), the articulation of research design is a particular benefit to archaeologists since it moves preparation to the less-fraught time before fieldwork begins. This preparatory work allows thoughtful development, testing, and revision of approaches, increasing the chances that the research design and collected data will support the desired outcomes. Finally, we recognize that exigencies of fieldwork may suggest a "pivot" due to an extraordinary find or the late discovery of unsuitability in approach or documentation during execution. Preparing for the unexpected again argues for careful a priori research design (e.g., consideration of abductive elements within deductive research), while the planning encouraged by preregistration should help to avoid errors in design (especially if combined with pilot research). Considering its potential value, the unpredictable nature of fieldwork should not preclude a "best-effort" at preregistration - and a "best-effort" in an abductive discipline like archaeology should be considered sufficient.

\section{Introducing Preregistration to Archaeological Practice}

Despite its potential utility, we could find no examples of rigorous preregistration in archaeology. A search of OSF's 304,904 registrations (as of March 19, 2020) produced only four non-teaching- 
related hits for "archaeology" (https://osf.io/registries/discover?provider=OSF\&q=archaeology). The OSF differentiates between categories of registration. The authors of the four preregistrations, Marwick (2017a, 2017c), Selden (2016), and Schmid (2019) used the "Open-Ended Registration" method to submit data and code in support of papers they had written. While the submission of code in support of scientific papers is laudable, these FAIR data "registrations", which support replication of computational results, do not represent the preregistration of approaches and methods we suggest.

Selden's pottery laser-scanning project (2017), for example, would be a good candidate for the preregistration of an inductive, postdictive project. A more complete preregistration effort, in this case, could include a protocol for the laser-scanning methodology and, crucially, a protocol for the creation and publication of metadata. Spot checks indicate that data documentation is incomplete; metadata like intra-site find location and artifact type do not accompany some scans (e. g. https://zenodo.org/search?q=41NA49).

Likewise, Marwick et al.'s lithic trampling experiment (2017a) represents an opportunity for the preregistration of more deductive, predictive research. This study explored, "claims of vertical movement of artifacts in debates surrounding the timing of the first human occupation," creating a physical experiment on the soil heaps of Madjedbebe (Marwick 2017a). Preregistration could articulate hypotheses, methodology, and an analytical approach. Perhaps, this preregistration could even include early versions of code. With moderate effort, especially if a grant application exists for this research, it could be elaborated to a full preregistration plan, or even a "registered report", in which a journal accepts or rejects the paper on the strength of its hypothesis, methodology, and proposed analysis before results are known (see Chambers 2019).

We note that Selden's presentation of the laser scan data with licensing information exceeds standard practice in archaeology, complements the associated paper (2017), and should be commended. Likewise, Marwick's publication of containerized code is exceptional, confirming his status as a leader in computational reproducibility. Rather, these examples highlight that (1) even archaeologists committed to transparency and reproducibility have not adopted comprehensive preregistration of approach, method, and data management, and (2) undertaking preregistration would reinforce other efforts to improve transparency and reproducibility, like FAIR datasets and publication of analytical code.

Templates, examples, and models could facilitate the introduction of preregistration into archaeology. Although a protocol for archaeological preregistration is beyond the scope of this chapter, and good practice around the execution of preregistration is still evolving (c.f. Nosek et al. 2018a; Ledgerwood 2018; Nosek et al. 2018b), we can offer some guidelines based on practices in other disciplines.

A distinction is sometimes made between preregistration of research questions or hypotheses on the one hand, and plans for analysis on the other:

"[P]reregistration of theoretical predictions helps researchers know how to correctly calibrate their confidence that a study tests (versus informs) a theory, whereas preregistration of analysis plans helps researchers know how to correctly calibrate their confidence that a specific finding is unlikely to be due to chance." (Ledgerwood 2018)

In the case of archaeology, experience with the FAIMS Project indicates that two additional aspects of archaeological research might also be registered profitably: data models and data 
workflows including data capture, manipulation, and analysis. Recording these aspects of research will help archaeologists meet emerging standards such as the TOP Level 2 Guidelines, which require "a full account of the procedures used to collect, preprocess, clean, or generate the data" (data provenance) and a "description of procedures" necessary for an "independent replication of the research" (Center for Open Science 2019). Thereby acknowledging that the abductive and serendipitous nature of the discipline may often make transparency the goal of preregistration, rather than replication. As noted above, the perfect should not be made the enemy of the good, and a best-effort attempt at preregistration would mark an improvement over the present lack of any at all.

Preregistration in archaeology could therefore include declarations of research tradition (e.g., theoretical framework), approach (e.g., inductive, deductive, idiographic, nomothetic, etc.), hypotheses or research questions, fieldwork and analysis plans, workflows, and/or data models. Compared to the current state of affairs, articulation of any aspect of research design would mark an improvement. An explicit commitment to a particular approach plus either hypotheses or research questions would do the most to combat researcher biases. Articulation of data models and workflows would do the most to overcome "just-in-time" fieldwork and produce reusable, widely comparable data at the end of the project.

In choosing a preregistration protocol, archaeologists will face a trade-off between the amount of time spent defining their approach and the resulting loss of flexibility. A "stricter" preregistration will maximize the benefits of preregistration, whereas a "looser" preregistration is faster and less constraining - but also less effective at avoiding bias or "just-in-time" fieldwork. A preregistration regime in archaeology could be built using existing knowledge infrastructures, like the Open Science Framework (Open Science Framework 2020; Bowman 2019). OSF provides a range of preregistration templates that could be adapted for archaeological use (Mellor and DeHaven 2016), including templates for "qualitative research" that accommodate the nature of archaeology as an abductive discipline while "providing a check on subjectivity" (Haven and Van Grootel 2019).

Archaeology would benefit from the development of domain-specific registration templates, such as that for Social Psychology (van 't Veer and Giner-Sorolla 2016), or perhaps for specific types of research, on the model of the "replication recipe" for conducting replication experiments in various disciplines (Brandt et al. 2014). We hope that the current chapter can inform the development of such templates, perhaps in consultation with the proprietors of archaeological data repositories like Open Context (see Kansa and Kansa 2010; Kansa and Bissell 2010), tDAR (McManamon and Kintigh 2016; McManamon et al. 2017), and the Archaeology Data Service, whose data dissemination and reuse expertise complements the field data management experience of the FAIMS Project.

Until archaeology-specific templates and protocols exist, we recommend starting with the OSF "Qualitative Research Preregistration" template (https://osf.io/6z2hr/; see Haven and Van Grootel 2019). It is intended for a range of social science research;not all fields are relevant to archaeology, and some archaeology-specific fields will probably need to be added. In our view, key fields from this template that are relevant to archaeology include:

- All "Study information" items (points 1-5), which provide basic project metadata and commitment to particular research questions should be included. "Typical moments" of research-question modification represents a useful concept that accommodates the emergent and serendipitous nature of archaeological research. As noted at 5.1, inductive 
vs. deductive should be indicated, and we would suggest adding idiographic vs. nomothetic. Projects with more of a deductive element should include any hypotheses that will be tested (5.2). Further information, such as the extent and nature of qualitative vs. quantitative analysis might also be useful. The "Use of Theory" section could likely be simplified and made more archaeology-specific.

- "Tradition" (Point 6) might be combined into the prior "Use of Theory" section, especially 5.3. This condensed section could include, the specific paradigm of fact-generation: "processualist," "post-processualist," "structuralist," "marxist," "cognitive archaeology," etc. (examples from Johnson 2010), The articulation of the paradigm used to generate facts may serve to contextualize the details of design, sampling, and analysis below.

- Regarding "Design Plan" (points 7-8), "Study Type" should be specified as "excavation," "surface survey", "artifact analysis", "geoarchaeology", etc., while "Study Design" is required for most grant or permit applications and could be generated from those sources. It would be useful to develop an archaeology-specific enumerated list of study types, and perhaps also of design elements linked to common approaches, rather than relying exclusively on a narrative study design.

- Under "Sampling Plan" (points 9-17), existing vs. non-existing data (and "Explanation of Existing Data" if relevant), "Data Collection Procedures" (adjusted for archaeology), "Type of Data Collected", "Sort of Sample", and "Data Collection Plan" should be specified. "Type of Sampling Rational" may be useful for quantitative or geospatial research. These categories might be modified or extended to ensure that the data model and data capture workflow are defined.

- "Script" (point 18) should read "Forms" (whether paper or digital) since it indicates "Data Collection instruments". Note again that modification during research is accommodated.

- An "Analysis Plan" (point 19) should be articulated, but with the categories at 19.1 amended to reflect archaeological practice. Important information might include the balance between quantitative and qualitative analysis, and/or a statement of the approach used for either type (e.g., the statistical approaches, application middle-range theory, etc.). It might also be useful to break the analytical procedures or workflow out from the descriptive section (19.2.1) into its own enumerated list.

- "Other" (point 20) provides a space for additional information that will help others to understand and reuse your research.

Beginning with a broad, existing template such as this one allows experimentation that, over time, can produce preregistration protocols that reflect and respect archaeological practice.

\section{Preregistration as "Slow Archaeology"}

The discipline of oceanography has also grappled with "small data" problems (Borgman 2015; Kansa and Bissell 2010), including diverse data and data structures that emerge from fieldwork, producing internally inconsistent datasets and mid-course changes to research. These problems include those that shade into questionable research practices, judging from Fraser et al.'s (Fraser et al. 2018) survey of related disciplines. As in archaeology, these problems inhibited 
the large-scale, synthetic research needed to address grand challenges in oceanography. In the course of aggregating and synthesizing data from many sources, the Ocean Health Index project discovered that:

"Environmental scientists are expected to work effectively with ever-increasing quantities of highly heterogeneous data even though they are seldom formally trained to do so... Without training, scientists tend to develop their own bespoke workarounds to keep pace, but with this comes wasted time struggling to create their own conventions for managing, wrangling and versioning data. If done haphazardly or without a clear protocol, these efforts are likely to result in work that is not reproducible - by the scientist's own 'future self' or by anyone else." (Lowndes et al. 2017).

The Ocean Health Index project's response to scalability and reproducibility was to implement data collection, manipulation, and analysis techniques based on open data formats, data standardization, and script-based analysis. Then, they created libraries of scripts that could perform commonly required analyses on well-structured data. The resulting data and associated analytical scripts could be shared, aggregated, and reused by researchers around the world. Increases in the quantity of available data and improving data quality were functions of reducing friction, avoiding technical debt, and implementing requirements around data formats, data structures, and analytical methods. More important than the generation of standardized, reusable data and scripts, however, was the thoughtful and planned research design, including its approach to creating, recording, and analyzing data.

Such careful planning not only aligns with the early processualists' call for more emphasis on thoughtful, a priori research design, but also with Caraher's recent argument for a "slow archaeology":

"Slow archaeology evokes the practice of archaeology as a craft. It prioritizes an embodied attentiveness to the entire process of fieldwork as a challenge to the fragmented perspectives offered by workflows influenced by our own efficient, industrialized age" (2015).

Preregistration and associated digital approaches that support transparency and reproducibility are not antithetical to slow archaeology - the chaos of "just-in-time" approaches to fieldwork are. Under-planned fieldwork might offer more freedom (and delay the day of reckoning with messy data), but it represents the opposite of the considered approach suggested by Caraher and is likely to result in biased and opaque research that does not reach its full potential. Preregistration encourages thoughtfulness.

\section{Conclusions}

Calls for archaeologists to improve data quality for large-scale research have been made for at least the past two decades (Doerr et al. 2004; Kansa and Kansa 2011; Faniel et al. 2013; Austin 2014), but limited progress has been made (Kintigh et al. 2014; Sobotkova 2018). Concerns over the transparency and reproducibility of archaeological research are more recent (Marwick 2017b), but archaeology is unlikely to avoid the reproducibility crisis so profoundly 
affecting other disciplines. Our recommendation to adopt preregistration as a means of increasing rigor is a pathway to solving those challenges. Preregistration promotes "built-in" rather than "bolt-on" good practice in research design, data management, and analysis. It likewise fosters thoughtful, "slow" archaeology rather than "just-in-time" archaeology. Robust, scalable, transparent, and reproducible results, underlying persuasive research directed at grand challenges, require planning and forethought. Making a public commitment to research design via preregistration, before setting out for the field, provides a mechanism that makes space for the necessary time and thought.

\section{References}

Adam, D. (2019). A solution to psychology's reproducibility problem just failed its first test. https://www.sciencemag.org/news/2019/05/solution-psychology-sreproducibility-problem-just-failed-its-first-test. Accessed: 2019-5-26.

Altschul, J. H., Kintigh, K. W., Klein, T. H., Doelle, W. H., Hays-Gilpin, K. A., Herr, S. A., Kohler, T. A., Mills, B. J., Montgomery, L. M., Nelson, M. C., Ortman, S. G., Parker, J. N., Peeples, M. A., and Sabloff, J. A. (2017). Opinion: Fostering synthesis in archaeology to advance science and benefit society. Proceedings of the National Academy of Sciences of the United States of America, 114(42):10999-11002.

Atici, L., Kansa, S. W., Lev-Tov, J., and Kansa, E. C. (2013). Other people's data: A demonstration of the imperative of publishing primary data. Journal of Archaeological Method and Theory, 20(4):663-681.

Austin, A. (2014). Mobilizing archaeologists: Increasing the quantity and quality of data collected in the field with mobile technology. Advances in Archaeological Practice, 2(1):13-23.

Baker, M. (2015). Smart software spots statistical errors in psychology papers. Nature News.

Ballsun-Stanton, B., Ross, S. A., Sobotkova, A., and Crook, P. (2018). FAIMS mobile: Flexible, open-source software for field research. SoftwareX, 7:47-52.

Borgman, C. L. (2015). Big data, little data, no data: scholarship in the networked world. MIT press.

Bowman, S. (2019). Understand registration forms. https://help.osf.io/hc/en-us/ articles/360019738794-Understand-Registration-Forms. Accessed: 2020-3-19.

Brandt, M. J., IJzerman, H., Dijksterhuis, A., Farach, F. J., Geller, J., Giner-Sorolla, R., Grange, J. A., Perugini, M., Spies, J. R., and van 't Veer, A. (2014). The replication recipe: What makes for a convincing replication? Journal of experimental social psychology, $50: 217-224$.

Caraher, W. (2015). Slow archaeology. North Dakota Quarterly, 80(2):43-52.

Center for Open Science (2018). Preregistration challenge. https://cos.io/prereg/. Accessed: 2018-5-15.

Center for Open Science (2019). TOP guidelines. https://cos .io/top/. Accessed: 2019-4-25. 
Chambers, C. (2019). What's next for registered reports? Nature, 573(7773):187-189.

Chase, J. M. (2013). The shadow of bias. PLoS biology, 11(7):e1001608.

DeHaven, A., Mellor, D., Haven, T., and van Grootel, L. (2019). Preregistration for qualitative research template (haven \& van grootel).

Derksen, M. (2019). Putting popper to work. Theory \&3 psychology, 29(4):449-465.

Doerr, M., Schaller, K., and Theodoridou, M. (2004). Integration of complementary archaeological sources. In Nicolucci, F. and Hermon, S., editors, Beyond the Artifact. Digital Interpretation of the Past. Proceedings of CAA2004, pages 64-69, Budapest. Archaeolingua.

Faniel, I., Kansa, E., Whitcher Kansa, S., Barrera-Gomez, J., and Yakel, E. (2013). The challenges of digging data: A study of context in archaeological data reuse. In Proceedings of the 13th ACM/IEEE-CS Joint Conference on Digital Libraries, JCDL '13, pages 295-304, New York, NY, USA. ACM.

Franco, A., Malhotra, N., and Simonovits, G. (2014). Social science. publication bias in the social sciences: unlocking the file drawer. Science, 345(6203):1502-1505.

Fraser, H., Parker, T., Nakagawa, S., Barnett, A., and Fidler, F. (2018). Questionable research practices in ecology and evolution. PloS one, 13(7):e0200303.

French, D. H. (1973). Theoretical and methodological aspects of data recovery. In Renfrew, C., editor, The Explanation of Culture Change: Models in Prehistory, pages 105-114. Duckworth, London.

GO-FAIR (2017). FAIR principles - GO FAIR. https://www.go-fair.org/fairprinciples/. Accessed: 2019-3-29.

Haven, T. L. and Van Grootel, L. (2019). Preregistering qualitative research. Accountability in research, 26(3):229-244.

Hole, F. (1973). Questions of theory in the explanation of culture change in prehistory. In Renfrew, C., editor, The Explanation of Culture Change: Models in Prehistory, pages 19-34. Duckworth, London.

Hollenbeck, J. R. and Wright, P. M. (2017). Harking, sharking, and tharking: Making the case for post hoc analysis of scientific data. Journal of management, 43(1):5-18.

Holub, P., Kohlmayer, F., Prasser, F., Mayrhofer, M. T., Schlünder, I., Martin, G. M., Casati, S., Koumakis, L., Wutte, A., Kozera, Ł., Strapagiel, D., Anton, G., Zanetti, G., Sezerman, O. U., Mendy, M., Valík, D., Lavitrano, M., Dagher, G., Zatloukal, K., van Ommen, G. B., and Litton, J.-E. (2018). Enhancing reuse of data and biological material in medical research: From FAIR to FAIR-Health. Biopreservation and biobanking, 16(2):97-105.

Huemer, M. (2020). You don't agree with karl popper. https://fakenous .net/?p=1239. Accessed: 2020-3-18.

Johnson, M. (2010). Archaeological Theory: An Introduction. John Wiley \& Sons. 
Kansa, E. C. and Bissell, A. (2010). Web syndication approaches for sharing primary data in“ small science" domains. Data Science Journal, 9:42-53.

Kansa, E. C. and Kansa, S. W. (2010). Publishing data in open context: Methods and perspectives. Center for the Study of Architecture Newsletter, 23(2).

Kansa, E. C. and Kansa, S. W. (2011). Toward a do-it-yourself cyberinfrastructure: Open data, incentives, and reducing costs and complexities of data sharing. In Kansa, E. C., Kansa, S. W., and Watrall, E., editors, Archaeology 2.0, volume 2, pages 57-92.

Kaplan, R. M. and Irvin, V. L. (2015). Likelihood of null effects of large NHLBI clinical trials has increased over time. PloS one, 10(8):e0132382.

Kerr, N. L. (1998). HARKing: hypothesizing after the results are known. Personality and social psychology review: an official journal of the Society for Personality and Social Psychology, Inc, 2(3):196-217.

Kintigh, K. (2006). The promise and challenge of archaeological data integration. American antiquity, 71(3):567-578.

Kintigh, K., Altschul, J. H., Beaudry, M. C., Drennan, R. D., Kinzig, A. P., Kohler, T. A., Limp, W. F., Maschner, H. D. G., Michener, W. K., Pauketat, T. R., Peregrine, P., Sabloff, J. A., Wilkinson, T. J., Wright, H. T., and Zeder, M. A. (2014). Grand challenges for archaeology. Proceedings of the National Academy of Sciences of the United States of America, 111(3):879-880.

Kuhn, T. S. (1970). The Structure of Scientific Revolutions (International Encyclopedia of Unified Science, Vol 1 and 2, Foundations of the Unity of Science: Vol. 2, No. 2). The University of Chicago Press, 2nd edition, enlarged edition.

Lakatos, I. (1978). Science and pseudoscience. BBC Radio Talk.

Ledgerwood, A. (2018). The preregistration revolution needs to distinguish between predictions and analyses. Proceedings of the National Academy of Sciences of the United States of America.

Lewis-Beck, M., Bryman, A. E., and Liao, T. F. (2003). The SAGE Encyclopedia of Social Science Research Methods. SAGE Publications.

Lowndes, J. S. S., Best, B. D., Scarborough, C., Afflerbach, J. C., Frazier, M. R., O'Hara, C. C., Jiang, N., and Halpern, B. S. (2017). Our path to better science in less time using open data science tools. Nature ecology \& evolution, 1(6):160.

Marwick, B. (2017a). Adaptations to sea level change and transitions to agriculture at khao toh chong rockshelter, peninsular thailand. https://osf.io/gved6. Accessed: 2020-3-19.

Marwick, B. (2017b). Computational reproducibility in archaeological research: Basic principles and a case study of their implementation. Journal of Archaeological Method and Theory, $24(2): 424-450$.

Marwick, B. (2017c). Movement of lithics by trampling: An experiment in the madjedbebe sediments. Open Science Framework. 
McManamon, F. and Kintigh, K. W. (2016). Making archaeological data and information discoverable, accessible, and usable for 21st century research: The theodore roosevelt dam archaeological project, tonto basin, arizona. Journal of Arizona Archaeology, 4(1):60-67.

McManamon, F. P., Kintigh, K. W., Ellison, L. A., and Brin, A. (2017). tDAR: A cultural heritage archive for Twenty-First-Century public outreach, research, and resource management. Advances in Archaeological Practice, 5(3):238-249.

McNutt, M., Lehnert, K., Hanson, B., Nosek, B. A., Ellison, A. M., and King, J. L. (2016). Liberating field science samples and data. Science, 351(6277):1024-1026.

Mellor, D. and DeHaven, A. (2016). Templates of OSF registration forms. https://osf .io/ zab38/. Accessed: 2020-3-19.

Mellor, D., Esposito, J., DeHaven, A., and Stodden, V. (2016). TOP resources - evidence and practices. https://osf.io/9f6gx/. Accessed: 2020-3-19.

Mons, B. (2020). Invest $5 \%$ of research funds in ensuring data are reusable. Nature, 578(7796):491.

Munafò, M. R., Nosek, B. A., Bishop, D. V. M., Button, K. S., Chambers, C. D., Percie du Sert, N., Simonsohn, U., Wagenmakers, E.-J., Ware, J. J., and Ioannidis, J. P. A. (2017). A manifesto for reproducible science. Nature Human Behaviour, 1:0021.

National Academies of Sciences, Engineering, and Medicine, Policy and Global Affairs, Committee on Science, Engineering, Medicine, and Public Policy, Board on Research Data and Information, Division on Engineering and Physical Sciences, Committee on Applied and Theoretical Statistics, Board on Mathematical Sciences and Analytics, Division on Earth and Life Studies, Nuclear and Radiation Studies Board, Division of Behavioral and Social Sciences and Education, Committee on National Statistics, Board on Behavioral, Cognitive, and Sensory Sciences, and Committee on Reproducibility and Replicability in Science (2019). Reproducibility and Replicability in Science. National Academies Press.

National Health and Medical Research Council (2018). The australian code for the responsible conduct of research. Technical Report R41, National Health and Medical Research Council.

National Health and Medical Research Council (2019). Open access policy. Technical report, National Health and Medical Research Council.

Nature (2016). Reality check on reproducibility. Nature, 533(7604):437.

NHMRC (2018). National statement on ethical conduct in human research. Technical Report E72, National Health and Medical Research Council.

Nosek, B. A., Alter, G., Banks, G. C., Borsboom, D., Bowman, S. D., Breckler, S. J., Buck, S., Chambers, C., Chin, G., Christensen, G., Contestabile, M., Dafoe, A., Eich, E., Freese, J., Glennerster, R., Goroff, D. L., Green, D., Hesse, B. W., Humphreys, M., Ishiyama, J., Karlan, D., Kraut, A., Lupia, A., Mabry, P. L., Madon, T., Malhotra, N., Mayo-Wilson, E., McNutt, M., Miguel, E., Paluck, E. L., Simonsohn, U., Soderberg, C. K., Spellman, B., Turitto, J., VandenBos, G., Vazire, S., Wagenmakers, E.-J., Wilson, R. K., Yarkoni, T., Stodden, V., and DeHaven, A. C. (2016). Transparency and openness promotion (TOP) guidelines. 
Nosek, B. A., Alter, G., Banks, G. C., Borsboom, D., Bowman, S. D., Breckler, S. J., Buck, S., Chambers, C. D., Chin, G., Christensen, G., Contestabile, M., Dafoe, A., Eich, E., Freese, J., Glennerster, R., Goroff, D., Green, D. P., Hesse, B., Humphreys, M., Ishiyama, J., Karlan, D., Kraut, A., Lupia, A., Mabry, P., Madon, T. A., Malhotra, N., Mayo-Wilson, E., McNutt, M., Miguel, E., Paluck, E. L., Simonsohn, U., Soderberg, C., Spellman, B. A., Turitto, J., VandenBos, G., Vazire, S., Wagenmakers, E. J., Wilson, R., and Yarkoni, T. (2015). Promoting an open research culture. Science, 348(6242):1422-1425.

Nosek, B. A., Ebersole, C. R., DeHaven, A. C., and Mellor, D. T. (2018a). The preregistration revolution. Proceedings of the National Academy of Sciences of the United States of America, 115(11):2600-2606.

Nosek, B. A., Ebersole, C. R., DeHaven, A. C., and Mellor, D. T. (2018b). Reply to ledgerwood: Predictions without analysis plans are inert. Proceedings of the National Academy of Sciences of the United States of America.

Open Science Collaboration (2015). Estimating the reproducibility of psychological science. Science, 349(6251):aac4716.

Open Science Framework (2020). OSF preregistration. https://osf.io/prereg/. Accessed: 2020-3-19.

Perkel, J. M. (2018). A toolkit for data transparency takes shape. Nature, 560(7719):513-515.

Ross, S., Ballsun-Stanton, B., Sobotkova, A., and Crook, P. (2015). Building the bazaar: Enhancing archaeological field recording through an open source approach. In Wilson, A. T. and Edwards, B., editors, Open Source Archaeology: Ethics and Practice, pages 111-129. De Gruyter Open, Warsaw, Poland.

Ross, S., Sobotkova, A., Ballsun-Stanton, B., and Crook, P. (2013). Creating eresearch tools for archaeologists: The federated archaeological information management systems project. Australian Archaeology, 77(1):107-119.

Rubin, M. (2017). When does HARKing hurt? identifying when different types of undisclosed post hoc hypothesizing harm scientific progress. Review of general psychology: journal of Division 1, of the American Psychological Association, 21(4):308-320.

Schmid, C. (2019). Research compendium for a contribution to 'human occupation and environmental change in the western maghreb during the last glacial maximum (LGM) and the late glacial. new evidence from the iberomaurusian site ifri el baroud (north-east morocco'). https://osf.io/e5c27. Accessed: 2020-3-19.

Selden, R. (2016). Ceramic asymmetry at the washington square mound site. http://dx.doi . org/10.17605/OSF. IO/M7ZGB.

Selden, R. Z. (2017). Asymmetry of caddo ceramics from the washington square mound site: An exploratory analysis. Digital Applications in Archaeology and Cultural Heritage, 5:21-28.

Snow, D. R., Gahegan, M., Giles, C. L., Hirth, K. G., Milner, G. R., Mitra, P., and Wang, J. Z. (2006). Cybertools and archaeology. Science, 311(5763):958. 
Sobotkova, A. (2018). Sociotechnical obstacles to archaeological data reuse. Advances in Archaeological Practice, 6(2):117-124.

Sobotkova, A., Ballsun-Stanton, B., Ross, S., and Crook, P. (2015). Arbitrary offline data capture on all of your androids: The FAIMS mobile platform. In Traviglia, A., editor, Across Space and Time. Papers from the 41st Annual Conference of Computer Applications and Quantitative Methods in Archaeology (CAA), pages 80-88, Perth. Amsterdam University Press.

Sobotkova, A., Ross, S. A., Ballsun-Stanton, B., Fairbairn, A., Thompson, J., and VanValkenburgh, P. (2016). Measure twice, cut once: Cooperative deployment of a generalized, Archaeology-Specific field data collection system. In Walcek Averett, E., Michael Gordon, J., and Counts, D. B., editors, Mobilizing the Past for a Digital Future: The Potential of Digital Archaeology, pages 337-371. The Digital Press @ University of North Dakota, Grand Forks, ND.

Stall, S., Yarmey, L., Cutcher-Gershenfeld, J., Hanson, B., Lehnert, K., Nosek, B., Parsons, M., Robinson, E., and Wyborn, L. (2019). Make scientific data FAIR. Nature, 570(7759):27.

Tavory, I. and Timmermans, S. (2014). Abductive Analysis: Theorizing Qualitative Research. University of Chicago Press.

van 't Veer, A. E. and Giner-Sorolla, R. (2016). Pre-registration in social psychology-a discussion and suggested template. Journal of experimental social psychology, 67:2-12.

VanValkenburgh, P., Silva, L. O. G., Repetti-Ludlow, C., Gardner, J., Crook, J., and BallsunStanton, B. (2018). Mobilization as mediation: Implementing a Tablet-Based recording system for ceramic classification. Advances in Archaeological Practice, pages 1-15.

Watson, P. J. (1973). Explanation and models: The prehistorian as philosopher of science and the prehistorian as excavator of the past. In Renfrew, C., editor, The Explanation of Culture Change: Models in Prehistory, pages 47-52. Duckworth, London.

Wilkinson, M. D., Dumontier, M., Aalbersberg, I. J., Appleton, G., Axton, M., Baak, A., Blomberg, N., Boiten, J.-W., da Silva Santos, L. B., Bourne, P. E., Bouwman, J., Brookes, A. J., Clark, T., Crosas, M., Dillo, I., Dumon, O., Edmunds, S., Evelo, C. T., Finkers, R., Gonzalez-Beltran, A., Gray, A. J. G., Groth, P., Goble, C., Grethe, J. S., Heringa, J., Hoen, P. A. C. t., Hooft, R., Kuhn, T., Kok, R., Kok, J., Lusher, S. J., Martone, M. E., Mons, A., Packer, A. L., Persson, B., Rocca-Serra, P., Roos, M., van Schaik, R., Sansone, S.-A., Schultes, E., Sengstag, T., Slater, T., Strawn, G., Swertz, M. A., Thompson, M., van der Lei, J., van Mulligen, E., Velterop, J., Waagmeester, A., Wittenburg, P., Wolstencroft, K., Zhao, J., and Mons, B. (2016). The FAIR guiding principles for scientific data management and stewardship. Scientific Data, 3:160018.

Yin, R. K. (2017). Case study research and applications: Design and methods. Sage publications. 


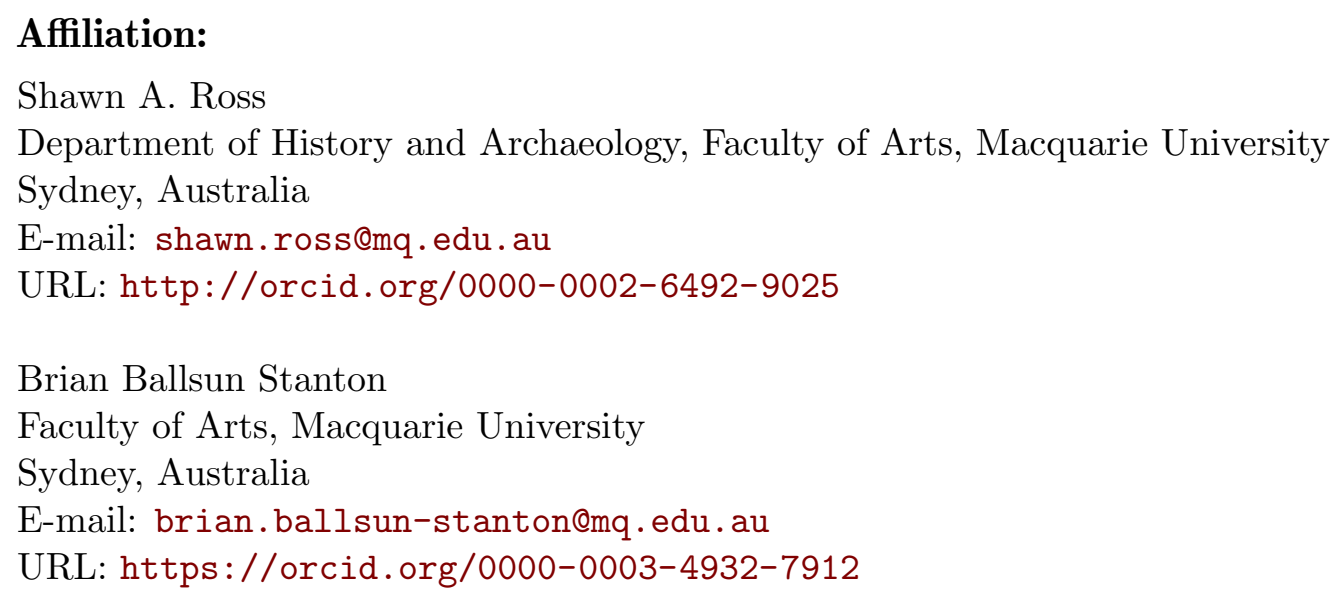

